\title{
The State-space Model of Micro-chaos
}

\author{
Gábor Csernák \\ HAS-BUTE \\ Research Group on Dynamics of Machines and Vehicles \\ Budapest, Hungary \\ csernak@mm.bme.hu
}

\author{
Gábor Stépán \\ Department of Applied Mechanics \\ Budapest University of Technology and Economics \\ Budapest, Hungary \\ stepan@mm.bme.hu
}

Received: July 28, 2019. Revised: October 10, 2021. Accepted: October 30, 2021. Published: November 23, 2021.

\begin{abstract}
Micro-chaos is the phenomenon when the sampling, the delay and the round-off lead to small amplitude chaotic oscillations in a digitally controlled system. It has been proved mathematically during the last few years in a couple of simple cases that the evolving vibrations are indeed chaotic. In this study, we partially generalize these results to the case when an originally unstable state of a system is stabilized by digital feedback control. It is pointed out that this type of systems are sensitive to initial conditions and there exists a finite attracting domain in their phase-space. We also show that the oscillations, related to micro-chaos may have a considerable influence on the accuracy and settling time of the control system. The application of numerical techniques is unavoidable in the case of chaotic systems. Several possibilities are highlighted in the paper for the numerical determination of important characteristics of microchaotic oscillations.
\end{abstract}

\section{INTRODUCTION}

The chaotic vibrations that are originated from the digital implementation of control are known for more than 20 years [1], [2], [3], and [4]. The consideration of the effects of sampling and processing delay is a routine procedure among control engineers [5], but the round-off in the analoguedigital converters is usually neglected. However, round-off frequently leads to deterministic - but small scale - chaotic behaviour which is often considered simply as stochastic noise in the practice. We think that certain properties of the control system could be improved by the proper modelling of the aforementioned deterministic effects.

Since the amplitude of chaotic vibrations, induced by the digital effects, is very small, this type of chaos is referred to as micro-chaos [3]. The chaotic nature of the oscillations was rigorously proved in Devaney's sense [7] in three simple cases: differential control without delay [3], differential control with delay [4], and PD control without delay [6]. According to Devaney's def nition of chaos, there must exist an attractor within which the solutions depend sensitively on initial conditions. Moreover, the map that governs the solutions must be topologically transitive and the periodic points must be dense in the attractor.

While the proof of the sensitive dependence on initial conditions and the existence of an attractor is quite simple in the considered cases, the proof of the fulf llment of the third and fourth conditions of chaos - topological transitivity and dense periodic points - is usually rather diff cult. Since the algorithms of the three aforementioned proofs are different, their generalization is not straightforward. In practice, the fulf llment of the frst two conditions already indicates the chaotic nature of the considered system. This is why systems with a positive Lyapunov exponent are usually considered chaotic.

The problems related to digital control gained attention in the felds of mathematics, control theory, and even neurobiology (see e.g. [8], [9], and [10], [11] and [12]). The communication among the scientists from these diverse felds is often diff cult due to the different formulation of similar problems. Our earlier papers about digital control focused only on mechanical models. In the present study, we generalize our results. The equations describing the effects of sampling and delayed feedback will be presented in Section 2, to introduce the notations used in the paper. While the publications in this f eld typically consider only small, toy-models, it is possibile to formulate the so-called micro-chaos map for full feedback systems of any size. This procedure will be shown in Section 3. In Section 4, the possibilities for the characterization of microchaotic oscillations are highlighted, via an example. The results are summarized in Section 5.

\section{STATE-SPACE MODEL OF FULL-STATE FEEDBACK WITH PROCESSING DELAY}

We consider systems that can be described in the continuous case (if there are no digital effects) by f rst order linear differential equations, i.e.,

$$
\dot{\mathbf{x}}=\tilde{\mathbf{A}} \mathbf{x}+\tilde{\mathbf{B}} u,
$$

where $\mathbf{x} \in \mathcal{R}^{n}$ denotes the state vector and $\tilde{\mathbf{A}} \in \mathcal{R}^{n \times n}$ and $\tilde{\mathbf{B}} \in \mathcal{R}^{n \times 1}$ are the coeff cient matrices. $u \in \mathcal{R}$ denotes the control signal that is a linear function of the state vector

$$
u(t)=\mathbf{K}_{0} \mathbf{x}(t) .
$$

In case of digital systems, the state of the system is known only at the sampling instants. It is typical in such cases that socalled zero-order-hold control is applied, i.e., the control signal is kept constant between the successive sampling instants. If some time is necessary for the calculation of the control signal, it will be the function of the previously sampled state (or states) of the system. We restrict ourselves to the cases when the delay is either zero or is an integer multiple of the sampling time. Thus, the control signal in the delayed case can be written as

$$
u_{i}=\mathbf{K}_{1} \mathbf{x}_{i-1}+\ldots+\mathbf{K}_{p} \mathbf{x}_{i-p}
$$

between the $i$ th and $i+1$ st sampling instants. Here $\mathbf{K}_{i} \in \mathcal{R}^{1 \times n}$ are the matrices of feedback gains. 


\section{A. The effect of sampling - without delay}

If there is no processing delay, the discretized form of the governing equations can be given as

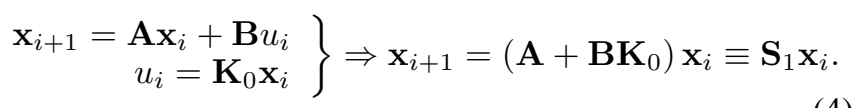

where the coeff cient matrices of the discrete system can be calculated as $\mathbf{A}=\exp (\tilde{\mathbf{A}} \tau)$ and $\mathbf{B}=\int_{0}^{\tau} \exp (\tilde{\mathbf{A}} z) d z \tilde{\mathbf{B}}, \mathbf{A} \in$ $\mathcal{R}^{n \times n}$ and $\mathbf{B} \in \mathcal{R}^{n \times 1}$,

If the $\mathbf{x}=\mathbf{0}$ state of the system is unstable without control, at least one of the eigenvalues of matrix $\mathbf{A}$ must be outside the unit circle on the complex plane. However, all the eigenvalues of matrix $\mathbf{S}_{1}$ must be inside the unit circle for the stabilization of the $\mathbf{x}=\mathbf{0}$ state.

\section{B. The effect of sampling - with processing delay}

If the control signal $u_{i}$ depends on $p$ pieces of the delayed values of the state, we arrive at

$$
\begin{aligned}
\mathbf{x}_{i+1} & =\mathbf{A} \mathbf{x}_{i}+\mathbf{B} u_{i}, \\
u_{i+1} & =\mathbf{K}_{1} \mathbf{x}_{i}+\mathbf{K}_{2} \mathbf{x}_{i-1}+\ldots+\mathbf{K}_{p} \mathbf{x}_{i-p+1} .
\end{aligned}
$$

By complementing the state vector $\mathbf{x}_{i}$ by the control signal $u_{i}$, a new state vector can be def ned $\mathbf{y}_{i}=\left[\begin{array}{llll}u_{i} & \mathbf{x}_{i} & \ldots & \mathbf{x}_{i-p+1}\end{array}\right]^{T}$ and the discrete map can be given in the form $\mathbf{y}_{i+1}=\mathbf{S}_{2} \mathbf{y}_{i}$.

$$
\left[\begin{array}{c}
u_{i+1} \\
\mathbf{x}_{i+1} \\
\vdots \\
\mathbf{x}_{i-p+2}
\end{array}\right]=\left[\begin{array}{cccc}
\mathbf{0} & \mathbf{K}_{1} & \ldots & \mathbf{K}_{p} \\
\mathbf{B} & \mathbf{A} & \mathbf{0} & \mathbf{0} \\
\mathbf{0} & \mathbf{I}_{1} & \mathbf{0} & \mathbf{0} \\
\mathbf{0} & \mathbf{0} & \mathbf{I}_{2} & \mathbf{0}
\end{array}\right]\left[\begin{array}{c}
u_{i} \\
\mathbf{x}_{i} \\
\vdots \\
\mathbf{x}_{i-p+1}
\end{array}\right]
$$

The sizes of the unit matrices are $\mathbf{I}_{1} \in \mathcal{R}^{n \times n}$ and $\mathbf{I}_{2} \in$ $\mathcal{R}^{n(p-1) \times n(p-1)}$. If $p=1$, these unit matrices do not show up in the hypermatrix. If $p=0$, there is no delay, thus, Equation (4) gives the correct form of the map, instead of (6). is

Thus, if there is no round-off, the general form of the map

$$
\mathbf{y}_{i+1}=\mathbf{S y}_{i},
$$

both with and without delay. $\mathbf{y}_{i} \equiv \mathbf{x}_{i} \in \mathcal{R}^{n}$ and $\mathbf{S} \equiv \mathbf{S}_{1}$, as def ned by (4) if there is no delay, while $\mathbf{y}_{i} \in \mathcal{R}^{p n+1}$ and $\mathbf{S} \equiv \mathbf{S}_{2}$, according to (6) in case of delay. We consider cases when the desired state of the continuous system is unstable without control, but stabilized by the control. Thus, all the eigenvalues of the corresponding matrix $\mathbf{S}$ must be less than one in modulus.

\section{POSSIBLE TYPES OF ROUND-OFF}

There are basically two analogue-digital transformations during the operation of a control system: on the one hand, the input state is measured and the measured values are quantized. On the other hand, the calculated control signal - the output can assume also only a f nite number of values. In the present section, these effects will be modelled. Note that we focus on the control system instead of the system that is controlled. This is why the state is referred to as input and the control signal is the output.

\section{A. Round-off at the output - without delay}

If there is no delay, no round-off at the input, but the calculated control signal $u_{i}$ is sent out with resolution $r_{O}$, the following map describes the evolution of the state instead of Equation (4):

$$
\begin{aligned}
\mathbf{x}_{i+1} & =\mathbf{A} \mathbf{x}_{i}+\mathbf{B} r_{O} \operatorname{Int}\left(\frac{u_{i}}{r_{O}}\right), \\
u_{i} & =\mathbf{K}_{0} \mathbf{x}_{i} .
\end{aligned}
$$

The function Int: $\mathcal{R} \rightarrow \mathcal{Z}$ calculates the integer part of the output, rounding towards zero. If the elements of the state vector $\mathbf{x}_{i}$ are small (e.g., $u_{i} / r_{O}=\mathbf{K}_{0} \mathbf{x}_{i} / r_{O}$ is less than one), the difference between the calculated and the rounded values of the output is rather large. Thus, the behaviour of the system is determined by the matrix $\mathbf{A}$, the system is locally unstable.

On the other hand, if the system's state is far from the origin, the effect of round-off is negligible. The large-scale behaviour of the system is well described if map (8) is rewritten as

$$
\mathbf{x}_{i+1}=\left(\mathbf{A}+\mathbf{B K} \mathbf{K}_{0}\right) \mathbf{x}_{i}-\mathbf{B} r_{O} f_{i}
$$

where $f_{i} \in \mathcal{R}$ denotes the neglected fractional part of the output, i.e., $f_{i} \in(-1,1), \forall i$. This fractional part does not inf uence considerably the behaviour of the system if the output value is large. Thus, the system is globally stable, since the matrix $\mathbf{S} \equiv \mathbf{S}_{1}=\mathbf{A}+\mathbf{B K}_{0}$ governs its large-scale behaviour.

\section{B. Round-off at the output - with delay}

If the delayed values of the state are used for the determination of the output, the map (corresponding to (6)) assumes the form

$$
\begin{gathered}
{\left[\begin{array}{c}
u_{i+1} \\
\mathbf{x}_{i+1} \\
\vdots \\
\mathbf{x}_{i-p+2}
\end{array}\right]=\left[\begin{array}{cccc}
\mathbf{0} & \mathbf{0} & \ldots & \mathbf{0} \\
\mathbf{B} & \mathbf{A} & \mathbf{0} & \mathbf{0} \\
\mathbf{0} & \mathbf{I}_{1} & \mathbf{0} & \mathbf{0} \\
\mathbf{0} & \mathbf{0} & \mathbf{I}_{2} & \mathbf{0}
\end{array}\right]\left[\begin{array}{c}
u_{i} \\
\mathbf{x}_{i} \\
\vdots \\
\mathbf{x}_{i-p+1}
\end{array}\right]} \\
+\left[\begin{array}{c}
r_{O} \operatorname{Int}\left(\sum_{j=1}^{p} \mathbf{K}_{j} \mathbf{x}_{i-j+1} / r_{O}\right) \\
\mathbf{0} \\
\mathbf{0}
\end{array}\right] \equiv \mathbf{U y}_{i}+\mathbf{b}_{i}, \\
\mathbf{0}
\end{gathered}
$$

where $\mathbf{U}$ is the coeff cient matrix of the uncontrolled system, with at least one eigenvalue outside the unit circle, according to our assumption. The alternative form of this map emphasizes the large-scale behaviour of the system:

$$
\begin{aligned}
{\left[\begin{array}{c}
u_{i+1} \\
\mathbf{x}_{i+1} \\
\vdots \\
\mathbf{x}_{i-(p-1)}
\end{array}\right] } & {\left[\begin{array}{cccc}
\mathbf{0} & \mathbf{K}_{0} & \ldots & \mathbf{K}_{p} \\
\mathbf{B} & \mathbf{A} & \mathbf{0} & \mathbf{0} \\
\mathbf{0} & \mathbf{I}_{1} & \mathbf{0} & \mathbf{0} \\
\mathbf{0} & \mathbf{0} & \mathbf{I}_{2} & \mathbf{0}
\end{array}\right]\left[\begin{array}{c}
u_{i} \\
\mathbf{x}_{i} \\
\vdots \\
\mathbf{x}_{i-p}
\end{array}\right] } \\
- & {\left[\begin{array}{c}
r_{O} f_{i} \\
\mathbf{0} \\
\mathbf{0} \\
\mathbf{0}
\end{array}\right] \equiv \mathbf{S y}_{i}-\mathbf{s}_{O} f_{i} . }
\end{aligned}
$$

Vectors $\mathbf{b}_{i}$ and $\mathbf{c}_{i} \equiv \mathbf{s}_{O} f_{i}$ contain the integer and fractional parts of the output signals, respectively. These vectors vary irregularly during the evolution of the system, leading to chaotic behaviour. 


\section{Round-off at the input}

If the measured inputs are quantized instead of the output, the maps corresponding to (8), (10) and (11) can be formulated as follows: if there is no delay,

$$
\begin{aligned}
\mathbf{x}_{i+1} & =\mathbf{A} \mathbf{x}_{i}+\mathbf{B K} \mathbf{r}_{I} \operatorname{Int}\left(\mathbf{r}_{I}^{-1} \mathbf{x}_{i}\right), \quad \text { and } \\
\mathbf{x}_{i+1} & =(\mathbf{A}+\mathbf{B K}) \mathbf{x}_{i}-\mathbf{B} \mathbf{K r}_{I} \mathbf{F}_{i},
\end{aligned}
$$

where the diagonal matrix

$$
\mathbf{r}_{I}=\operatorname{diag}\left[\begin{array}{llll}
r_{I 1} & r_{I 2} & \ldots & r_{I n}
\end{array}\right]
$$

contains the values of the resolution for the $n$ state variables. The function Int: $\mathcal{R}^{n} \rightarrow \mathcal{Z}^{n}$ calculates the integer part of its operand. The neglected fractional parts of the state vectors are collected to vector $\mathbf{F}_{i}$

In case of delay, the coeff cient matrices are the same as in Eqs. (10) and (11), and the shift vectors can be given as

$$
\begin{gathered}
\mathbf{b}_{i}=\left[\begin{array}{c}
\sum_{j=1}^{p} \mathbf{K}_{j} \mathbf{r}_{I} \mathbf{I n t}\left(\mathbf{r}_{I}^{-1} \mathbf{x}_{i-j+1}\right) \\
\mathbf{0} \\
\mathbf{0} \\
\mathbf{0}
\end{array}\right], \\
\mathbf{s}_{i}=\left[\begin{array}{c}
\sum_{j=1}^{p}\left(\mathbf{K}_{j} \mathbf{r}_{I} \mathbf{F}_{i-j+1}\right) \\
\mathbf{0} \\
\mathbf{0} \\
\mathbf{0}
\end{array}\right]=\left[\begin{array}{c}
\sum_{j=1}^{p}\left(\mathbf{K}_{j}\right) \mathbf{r}_{I} \tilde{\mathbf{F}}_{i} \\
\mathbf{0} \\
\mathbf{0} \\
\mathbf{0}
\end{array}\right] .
\end{gathered}
$$

Note that the fractional part vectors $\mathbf{F}_{i-j+1}, j=1 \ldots p-$ each having elements between -1 and $1-$ can be exchanged here by a single vector $\tilde{\mathbf{F}}_{i}$ if the corresponding elements of vectors $\mathbf{K}_{j}$ have the same sign. Othervise, the absolute values of the elements must be considered.

Thus, we found that independently on the type of roundoff, the discrete map describing the evolution of the system can be given as $\mathbf{y}_{i+1}=\mathbf{U y}_{i}+\mathbf{b}_{i}$ or $\mathbf{y}_{i+1}=\mathbf{S y}_{i}-\mathbf{c}_{i}$. We will refer to these maps as micro-chaos maps.

\section{CHARACTERIZATION OF MICRO-CHAOS}

Several quantities were introduced in the theory of dynamical systems for the characterization of chaotic behaviour. In the present section, we calculate some of these quantities in the case of a realistic example, using algorithms that best suit to the considered problem. Our main goal is to $\mathrm{f}$ nd those properties of micro-chaos that may inf uence the macro-scale behaviour of the controlled system.

\section{A. Size of the attracting domain}

1) Existence of an attracting domain: From the practical point of view, the most important characteristics of microchaotic behaviour are the expected amplitude of the oscillations and the maximal distance of the attractor from the desired state. These two distances are approximately equal if there is only one attractor in the state-space, in the neighbourhood of the desired state. However, it was found [4], [13], that their values may differ by several orders of magnitude if disconnected attractors coexist. Even in the latter case, the attractors can be found in a f nite domain, in a so-called absorbing sphere. To estimate the size of this sphere, we rewrite the map

$$
\begin{array}{r}
\mathbf{y}_{i+1}=\mathbf{S} \mathbf{y}_{\mathbf{i}}-\mathbf{c}_{i} \quad \text { as } \\
\mathbf{y}_{i+1}=\mathbf{S}^{i} \mathbf{y}_{0}-\sum_{j=0}^{i-1} \mathbf{S}^{j} \mathbf{c}_{j},
\end{array}
$$

The maximal possible norm $\left|\mathbf{y}_{\infty}\right|=\lim _{i \rightarrow \infty} \max \left|\mathbf{y}_{i}\right|$ of the vectors $\mathbf{y}_{i}$ provides an estimation for the size of the attracting domain at the origin.

Unfortunately, matrix $\mathbf{S}$ is not a so-called normal matrix, i.e., its norm (the greatest singular value) can be larger than 1. Still - since $\mathbf{S}$ is chosen such that its eigenvalues are inside the unit circle -

$$
\lim _{i \rightarrow \infty}\left\|\mathbf{S}^{i}\right\|=0
$$

is fulf lled [4]. Thus,

$$
\left|\mathbf{y}_{\infty}\right|=\max \left|\sum_{j=0}^{\infty} \mathbf{S}^{j} \mathbf{c}_{j}\right| .
$$

The details of the application of this formula depend on the properties of matrix $\mathbf{S}$ and vector $\mathbf{c}_{j}$.

- In most of the cases, $\mathbf{S}$ is diagonalizable. In this case, $\mathbf{S}$ can be expressed as a diagonal matrix $\hat{\mathbf{S}}$ in the basis of eigenvectors. Let $\mathbf{T}$ denote the (in general complexvalued) matrix that is composed of the eigenvectors of S. By multiplying both sides of (16) by $\mathbf{T}^{-1}$ and formally taking the limit $i \rightarrow \infty$, one obtains

$$
\mathbf{T}^{-1} \mathbf{y}_{\infty}=\sum_{j=0}^{\infty} \mathbf{T}^{-1} \mathbf{S}^{j} \mathbf{T} \mathbf{T}^{-1} \mathbf{c}_{j} .
$$

Consider the case when the output signal is rounded. In this case, vectors $\mathbf{c}_{j}$ can be expressed as $\mathbf{c}_{j}=\mathbf{s}_{O} f_{j}$ (cf. (9)). In the basis of the eigenvectors of $\mathbf{S}$,

$$
\mathbf{T}^{-1} \mathbf{c}_{j}=\left[\begin{array}{lll}
\tilde{s}_{O 1} & \ldots & \tilde{s}_{O n}
\end{array}\right]^{T} f_{j} .
$$

Thus,

$$
\mathbf{T}^{-1} \mathbf{y}_{\infty}=\left[\begin{array}{c}
\sum_{j=0}^{\infty} \lambda_{1}^{j} \tilde{s}_{O 1} f_{j} \\
\vdots \\
\sum_{j=0}^{\infty} \lambda_{n}^{j} \tilde{s}_{O n} f_{j}
\end{array}\right] .
$$

If the eigenvalues $\lambda_{k}$ are complex or negative real numbers, the choice $f_{j}=1, \forall j$ does not lead to the maximal norm of $\mathbf{y}_{\infty}$. This problem can be overcome if the absolute values of the eigenvalues and the elements of $\mathbf{s}_{O}$ and $\mathbf{T}$ are considered:

$$
\left|\mathbf{y}_{\infty}\right|<\sum_{i=1}^{n}\left|\sum_{j=1}^{n}\right| T_{i j}\left|\frac{\left|\tilde{s}_{O j}\right|}{1-\left|\lambda_{j}\right|}\right| .
$$

- In the rare case, when $\mathbf{S}$ is not diagonalizable, further results can be used from the theory of non-normal matrices [14]. Unfortunately the upper estimates that are based on the general theory usually provide unrealistically large numbers. These large numbers cannot 
tbe used for the prediction of the behaviour of the real control system.

$\left|\mathbf{y}_{\infty}\right|$ is necessarily f nite, since the eigenvalues of $\mathbf{S}$ are inside the unit circle. The generalization of formula (22) is straightforward. Thus, we can claim that there exists a f nite attracting domain for each version of the micro-chaos map in the neighbourhood of the origin. Note, that if the size of the attracting domain is to be determined in a practical problem, the state variables must be rescaled to dimensionless form. If the variables are displacement, angle, speed and angular velocity, this step can be done by rescaling the time and the distance.

2) Example: To illustrate the result of this section via an example, consider the problem of the inverted, PD-controlled pendulum, without processing delay. In this case, the variation of the angle $\varphi$ and the angular velocity $\omega$ of the pendulum can be given by the following equations between two successive sampling instants $t \in[i \tau,(i+1) \tau)$ :

$$
\begin{aligned}
& \dot{\varphi}=\omega, \\
& \dot{\omega}=\alpha^{2} x-\left(P \varphi_{i}+D \omega_{i}\right) .
\end{aligned}
$$

The corresponding linear two-dimensional map assumes the form

$$
\left[\begin{array}{l}
\varphi_{i+1} \\
\omega_{i+1}
\end{array}\right]=\left[\begin{array}{cc}
\operatorname{ch}-P \frac{\mathrm{ch}-1}{\alpha^{2}} & \frac{\mathrm{sh}}{\alpha}-D \frac{\mathrm{ch}-1}{\alpha^{2}} \\
\alpha \operatorname{sh}-P \frac{\mathrm{sh}}{\alpha} & \operatorname{ch}-D \frac{\mathrm{sh}}{\alpha}
\end{array}\right]\left[\begin{array}{l}
\varphi_{i} \\
\omega_{i}
\end{array}\right],
$$

where $\operatorname{ch} \equiv \cosh (\alpha \tau)$, and $\operatorname{sh} \equiv \sinh (\alpha \tau)$. Thus, using the notations, introduced in the previous sections, $\mathbf{x}_{i}=\operatorname{col}\left[\begin{array}{ll}\varphi_{i} & \omega_{i}\end{array}\right]$ and

$$
\mathbf{A}=\left[\begin{array}{cc}
\mathrm{ch} & \frac{\mathrm{sh}}{\alpha} \\
\alpha \mathrm{sh} & \mathrm{ch}
\end{array}\right], \quad \mathbf{B}=\left[\begin{array}{c}
\frac{\mathrm{ch}-1}{\alpha^{2}} \\
\frac{\mathrm{sh}}{\alpha}
\end{array}\right], \quad \mathbf{K}=\left[\begin{array}{c}
-P \\
-D
\end{array}\right] .
$$

The coeff cient matrix in (24) is $\mathbf{S} \equiv \mathbf{A}+\mathbf{B K}$. It can be checked by Jury's criterions [6] that its eigenvalues are inside the unit circle if

$$
\begin{aligned}
& P>a_{2 \text { cond }} \equiv \alpha^{2} \\
& D<a_{0 \text { cond }} \equiv \frac{\alpha(1+\cosh (\alpha \tau))}{\sinh (\alpha \tau)} \\
& D>a_{1 \text { cond }} \equiv \frac{\cosh (\alpha \tau)-1}{\alpha \sinh (\alpha \tau)} P .
\end{aligned}
$$

Thus, the domain of stability forms a triangle on the $P$ $D$ parameter plane. We restrict ourselves to this parameter domain. Note that the corresponding stability domain of a similar system with viscous damping and delay was derived in [13].

Before applying formula (22), the state variables must be rescaled. By introducing the dimensionless time $T=t / \tau$, dimensionless variables $\hat{\varphi}=\varphi /\left(\tau^{2} r_{O}\right), \hat{\omega}=\omega /\left(\tau r_{O}\right)$, and parameters $\hat{\alpha}=\alpha \tau, \hat{P}=P \tau^{2}$, and $\hat{D}=D \tau$, the equations become dimensionless. We used the parameters that correspond to a real inverted pendulum [15]: $\hat{\alpha}=6.8511 \cdot 10^{-2}$, $\hat{P}=5.4997 \cdot 10^{-3}$ and $\hat{D}=2.5665 \cdot 10^{-2}$. In the remaining part of the paper, the hat symbols of dimensionless quantities will be discarded. There are altogether 12 disconnected attractors in the phase-space of the system. In Figs. 1 and 2 two local attractors are shown. The size of the global attracting domain was estimated by formula (22) and also by numerical

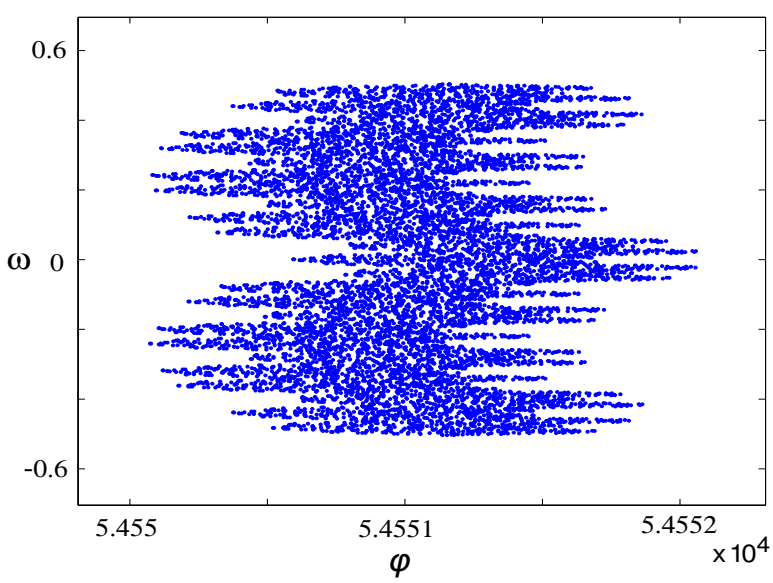

Fig. 1. An attractor of the system.

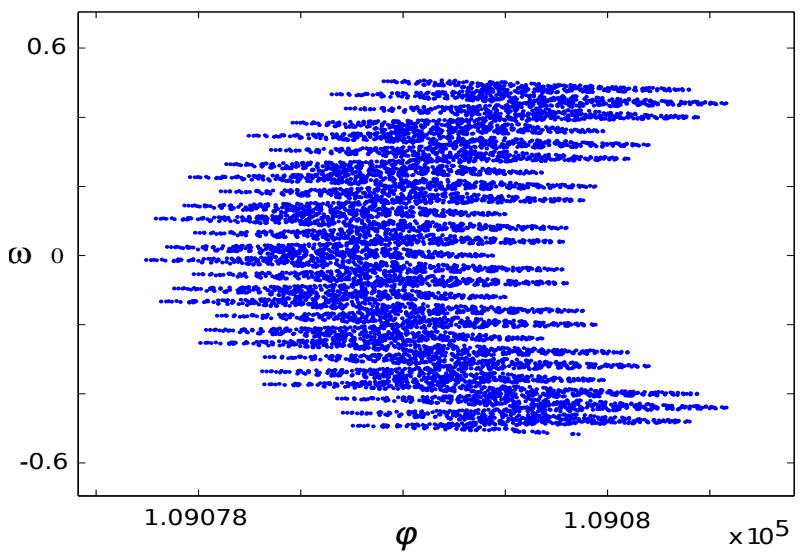

Fig. 2. The attractor farthest from the origin.

experiments. The results, together with the imaginary part of one of the eigenvalues of $\mathbf{S}$ are depicted in a logarithmic scale in Fig. 3 as function of parameter $P$. If parameter $D$ is increased, the eigenvalues of $\mathbf{S}$ become real. Consequently, the estimate (22) becomes more accurate, see Fig. 4.

\section{B. Sensitivity to initial conditions}

It is quite trivial that the eigenvalues of matrix $\mathbf{U}$ are the Lyapunov exponents of the system. In the considered example, $\lambda_{1,2}=\exp ( \pm \hat{\alpha})$. Note that the Lyapunov exponents of the map and the underlying fow are related as $\lambda_{\text {flow }}=\lambda_{\text {map }} / \tau$. For the dimensionless case, these exponents are equal. One of the greatest diff culties during the numerical simulation of the map is the estimation of the duration of transients. Because several attractors coexist, the solution may spend quite a long time in the neighbourhood of a chaotic attractor, before reaching another attractor. The exact value of the largest Lyapunov exponent is known: $\lambda_{\max }=\exp (\hat{\alpha}) \approx 1.00687$. Thus, the rate of convergence and the error of a numerical Lyapunov exponent estimation method can be determined and used for the estimation of the properties of the transient behaviour of the system. We determined the Lyapunov exponent by a method described in [16]. The results are shown in Fig. 5. As it can be seen, the error of the Lyapunov exponent calculation is indeed 


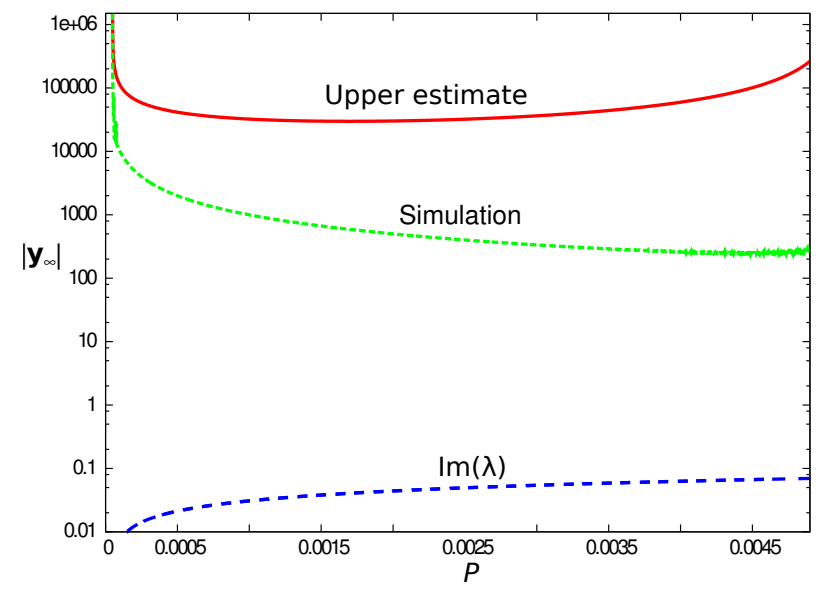

Fig. 3. The estimated and the numerically determined size of the global attractor of the system, together with the variation of the imaginary part of the eigenvalues (the real part and the norm of the matrix practically do not change).

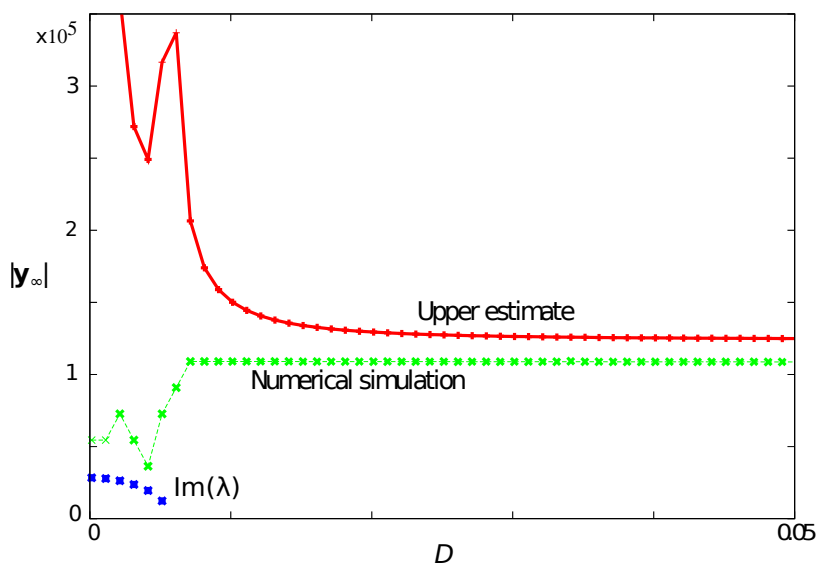

Fig. 4. The estimated and the numerically determined size of the global attractor of the system

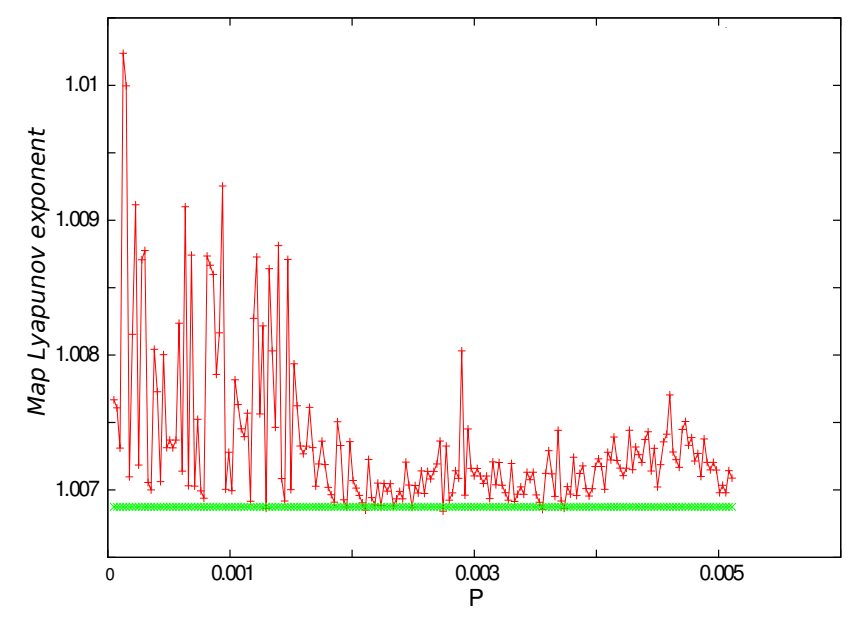

Fig. 5. The correct Lyapunov exponent and its estimated value. larger if the size of the attracting domain is also large - cf. Fig. 3 .

\section{Dimension of the attractor}

The number of possible attractors within the global attracting domain is hard to determine automatically. In order to explore the structure of local attractors, we performed several numerical simulations with varying initial conditions and detected the positions of the middle points of the resulting attractors. As Fig. 6 shows, these points take place according to a certain pattern. The fractal dimension $D_{0}$ of the attractors

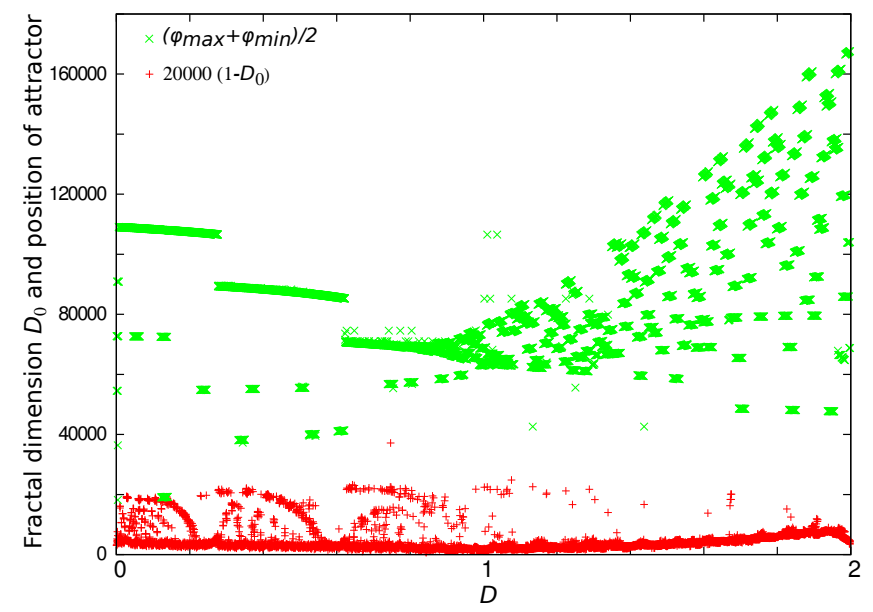

Fig. 6. The fractal dimension of the local attractors and their position.

was also calculated. The results are also depicted in the fgure. Although we implemented only a rather simple automatic boxcounting algorithm, it is easy to see that the fractal dimension changes in accordance with the appearance of new attractors. We hope that by applying a ref ned calculation method, we will be able to characterize the local attractors more accurately.

\section{Periodic points}

The periodic points of micro-chaos maps of the form $\mathbf{y}_{i+1}=\mathbf{U y}_{i}+\mathbf{b}_{i}$ can be found simply according to the def nition of periodic points, i.e., that after $p$ iteration steps, the state vector becomes equal to the initial vector: $\mathbf{y}_{\mathbf{p}}=\mathbf{y}_{\mathbf{0}}$. This form of the map can be rewritten similarly to (16). Thus, using the notation $\mathbf{b}_{i}=\mathbf{G} m_{i}$ - where $m_{i}, i=0 \ldots p-1$ are the integer parts of the output at the subsequent steps -, the initial state of a possible $p$-periodic solution can be expressed as

$$
\mathbf{y}_{\mathbf{0}}=\left(\mathbf{I}-\mathbf{U}^{p}\right)^{-1}\left(\mathbf{U}^{p-1} \mathbf{G} m_{0}+\ldots \mathbf{U}^{0} \mathbf{G} m_{p-1}\right) .
$$

Unfortunately, the results must be checked for the possible combinations of the integer numbers $m_{i}$, which is very time consuming. We performed this task in the case of the considered example for periods 1-6. The results are presented in Fig. 7. At the parameters of the example, the farthest 6 periodic point is at $\varphi \approx 106500$, while the farthest attractor is at $\varphi \approx 109080$. In general, the 6 periodic points are not in the closures of the local attractors, but they are in the global attracting domain. Thus, a lower estimate of the size of the attracting domain can be obtained, based on this calculation. 


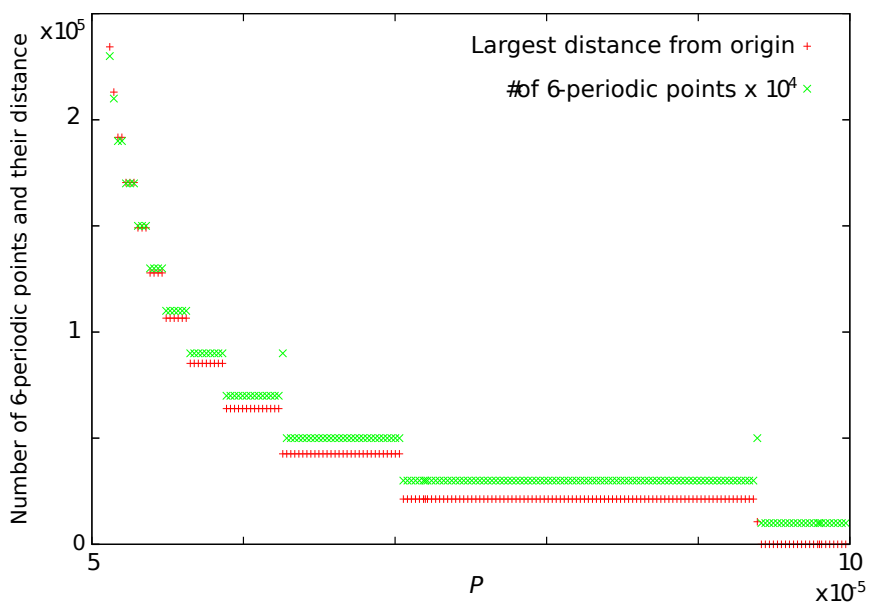

Fig. 7. The number of 6 periodic points and their maximal distance from the origin-

\section{SUMMARY}

We generalized the so-called micro-chaos maps for fullstate feedback systems of arbitrary dimension. Two types of round-off were taken into account; the resulting maps have similar structure. A formula was derived for the estimation of the largest possible round-off-induced control error and three different possibilities were highlighted for the characterization of micro-chaotic behaviour: 1) since the Lyapunov exponent is known, the transients of the chaotic motion can be fltered out by checking the convergence properties of Lyapunov exponent calculation algorithms. 2) Because several local attractors may co-exist, the exploration of all of these attractors is rather diff cult numerically. Our latest results indicate that the fractal dimension of a numerically obtained attractor may be used as an indicator of bifurcations that give rise to new attractors. 3) The positions of periodic points can be used for giving a lower estimate of the size of the global attractor.

\section{ACKNOWLEDGMENTS}

This research was supported by the Hungarian National Science Foundation under grant no. OTKA K 83890.

\section{REFERENCES}

[1] G. Chen, X. Dong, From Chaos to Order: Methodologies, Perspectives and Applications, World Scientif c, Singapore, 1998.

[2] F.D. Delchamps, Stabilizing a linear system with quantized state feedback, IEEE Trans. Autom. Contr., 35 pp. 916-924, 1990.

[3] Haller, G. - Stépán, G.: Micro-Chaos in Digital Control, J. Nonlinear Sci., 6, pp. 415-448, 1996.

[4] Csernák, G. - Stépán, G.: Digital Control as Source of Chaotic Behavior, International Journal of Bifurcation and Chaos, 20(5), pp. 1365-1378, 2010.

[5] B.C. Kuo, Digital Control Systems, SRL Publishing, Champaign, IL, USA, 1977.

[6] G. Csernák, G. Stépán: Sampling and Round-off, as Sources of Chaos in PD-controlled Systems, Proceedings of the 19th Mediterranean Conference on Control and Automation, June 20-23, 2011, Corfu

[7] R.L. Devaney, An Introduction to Chaotic Dynamical Systems, AddisonWesley, 1989.

[8] M. Landry, S.A. Campell, K. Morris and C.O. Aguilar, Dynamics of an inverted pendulum with delayed feedback control, SIAM Journal on Applied Dynamical Systems 4(2) pp. 333-351, 2005.
[9] J. Sieber, B. Krauskopf, Complex Balancing Motions of an Inverted Pendulum Subject to Delayed Feedback Control, Physica D 197(3-4), pp. 332-345, 2004.

[10] R. Kelly, V. Santibanez, A. Loria, Control of Robot Manipulators in Joint Space, Springer, London, 2005.

[11] A.H.C. Gosline, V. Hayward, H. Michalska, Ineluctability of Oscillations in Systems with Digital Implementation of Derivative Feedback, Automatica, 47(11), pp. 2444-2450, 2011.

[12] A. Potapov, M.K. Ali, Chaotic Neural Control, Physical Review E, 63(4), 046215, 2001.

[13] G. Csernák, G. Stépán, Disconnected Chaotic Attractors in Digitally Controlled Linear Systems, Recent Researches in Automatic Control, Systems Science and Communications, Proceedings of the 8th WSEAS International Conference on Dynamical Systems and Control, Published by WSEAS Press, ISBN: 978-1-61804-103-6, pp. 97-102, 2012.

[14] Lloyd Nicholas Trefethen, Mark Embree, Spectra And Pseudospectra: The Behavior of Nonnormal Matrices And Operators, Princeton University Press, 2005

[15] E. Enikov, G. Stépán, Micro-Chaotic Motion of Digitally Controlled Machines, J. of Vibration and Control, 4, pp. 427-443, 1998.

[16] Andrzej Stefanski, Tomasz Kapitaniak, Estimation of the dominant Lyapunov exponent of non-smooth systems on the basis of maps synchronization, Chaos, Solitons and Fractals 15, pp. 233-244, 2003.

\section{Creative Commons Attribution License 4.0 (Attribution 4.0 International, CC BY 4.0)}

This article is published under the terms of the Creative Commons Attribution License 4.0

https://creativecommons.org/licenses/by/4.0/deed.en_US 